Slavica

bruxellensia

\section{Slavica bruxellensia}

Revue polyphonique de littérature, culture et histoire

slaves

$11 \mid 2015$

Littérature et philosophie

\title{
Sur un débat qui n'a pas eu lieu : approche ingardénienne contre approche meinongienne des objets fictifs
}

\section{Sébastien Richard}

\section{(2) OpenEdition}

\section{Journals}

\section{Édition électronique}

URL : http://journals.openedition.org/slavica/1719

DOI : 10.4000/slavica.1719

ISSN : 2034-6395

\section{Éditeur}

Université libre de Bruxelles - ULB

\section{Référence électronique}

Sébastien Richard, «Sur un débat qui n'a pas eu lieu : approche ingardénienne contre approche meinongienne des objets fictifs », Slavica bruxellensia [En ligne], 11 | 2015, mis en ligne le 15 janvier 2015, consulté le 20 avril 2019. URL : http://journals.openedition.org/slavica/1719 ; DOI : 10.4000/ slavica. 1719

Ce document a été généré automatiquement le 20 avril 2019.

\section{c) () (위}

Les contenus de Slavica bruxellensia sont mis à disposition selon les termes de la Licence Creative Commons Attribution - Pas d'Utilisation Commerciale - Pas de Modification 3.0 France. 


\title{
Sur un débat qui n'a pas eu lieu: approche ingardénienne contre approche meinongienne des objets fictifs
}

\author{
Sébastien Richard
}

\section{Introduction}

1 Alexius Meinong et Roman Ingarden appartiennent tous deux à ce que l'on appelle l'École de Brentano, le premier directement, puisqu'il fut l'un des élèves de Franz Brentano à Vienne, et le second indirectement, puisqu'il fut l'élève de Kazimierz Twardowski et d'Edmund Husserl, eux-mêmes élèves de Brentano. Ingarden peut d'autant plus être rattaché à cette tradition philosophique autrichienne qu'il fut l'un des disciples les plus enthousiastes de la philosophie dite réaliste développée par Husserl dans ses Logische Untersuchungen (Recherches logiques, 1900-1901), mais n'a jamais accepté le tournant transcendantal de son maître opéré officiellement avec la publication des Idées directrices pour une phénoménologie pure et une philosophie phénoménologique (Ideen I).

Étant donné cette appartenance à une même tradition, il n'est pas étonnant que les philosophies de Meinong et d'Ingarden se rencontrent sur de nombreux points. C'est en particulier le cas en ce qui concerne la fiction. Dans cet article, nous procéderons à une comparaison des approches meinongienne et ingardénienne de ces objets particuliers, souvent qualifiés de non existants, que sont les objets fictifs. Nous nous intéresserons ici uniquement aux objets fictifs issus des œuvres d'art littéraires, ceux que l'on trouve dans les romans, les nouvelles, les drames ou les poèmes lyriques. Sherlock Holmes et Hamlet sont des objets fictifs en ce sens, mais pas la planète Vulcain postulée par Urbain Le Verrier. 
3 Si elle n'est pas neuve ${ }^{1}$, il faut tout de même souligner que la confrontation des approches ingardénienne et meinongienne des objets fictifs est elle-même largement fictive : elle relève plus de la reconstruction théorique que de la recherche historico-philosophique. En effet, outre qu'ils n'ont jamais directement débattu philosophiquement l'un avec l'autre de questions relatives aux objets fictifs, que ce soit dans des publications officielles ou par correspondance, il n'y a à strictement parler pas de théorie meinongienne de la fiction. Comme le remarque Venanzio Raspa, « Meinong n'a jamais écrit un seul livre ou article sur l'esthétique ou l'ontologie des œuvres d'art, ni fourni un système structuré permettant de traiter les objets fictifs esthétiques $\|^{2}$. C'est dès lors un petit paradoxe que de constater que le «meinongianisme » est l'une des approches les plus répandues dans la philosophie contemporaine de la fiction 3 . Celle-ci est en fait avant tout l'œuvre d'auteurs qui, comme Terence Parsons, Richard Routley, Edward Zalta, William Rapaport, Dale Jacquette, ou encore Jacek Paśniczek ${ }^{4}$, ont puisé à partir des années 1970 leur inspiration dans la «théorie de l'objet» de Meinong pour développer, au moyen des outils logiques modernes, des logiques dites "néomeinongiennes", pouvant, entre autres, s'appliquer aux objets fictifs. Pour reconstruire l'approche meinongienne de la fiction, nous nous appuierons dans ce qui suit avant tout sur les écrits de Meinong lui-même, mais lorsque cela s'avérera intéressant, nous n'hésiterons pas à la compléter en nous appuyant sur certains travaux de l'École néomeinongienne.

Meinong et Ingarden partagent la conviction fondamentale suivant laquelle il y a des objets fictifs. Nos deux philosophes s'opposent donc à toute tentative réductionniste qui soutient, à l'instar de Bertrand Russell, qu'il n'y a pas d'objets de ce type, parce que nous pouvons substituer à toute phrase contenant un terme faisant référence à un objet fictif une paraphrase qui en est dépourvue. Mais il ne suffit pas d'affirmer simplement qu'il y a des objets fictifs, encore faut-il préciser leur mode d'être, du moins s'ils en possèdent un.

\section{Modes d'être et objets fictifs selon Meinong et Ingarden}

Dans un texte de 1905, Theodor Lipps écrivait :

Je sais que le Méphistophélès de Goethe est une forme poétique pure, qu'il n'y a jamais eu de Méphistophélès, que donc il n'a jamais énoncé aucun des mots que Goethe lui fait dire. Néanmoins, je peux discuter de la manière dont Méphistophélès répond à Faust ou au Seigneur dans certains passages. Je peux dire qu'il répond « de fait » ainsi, et pas autrement.

(...) D'un autre côté, je ne parle pas du tout du Méphistophélès historique, mais plutôt de celui de Goethe, ou plus précisément du Méphistophélès de l'histoire. Mais celui-ci a un mode d'existence caractéristique. Il ne fait aucun doute qu'il a autrefois été créé par Goethe. Mais ayant été créé et faisant l'objet d'une représentation artistique dans un texte littéraire, il possède une sorte de réalité. Sans aucun doute, ce qu'il fait et ce qu'il dit sont en un certain sens des « faits » que nous pouvons constater. ${ }^{5}$

Lipps qualifie le type de réalité dont jouit ici Méphistophélès de "réalité esthétique » ( ästhetische Wirklichkeit). Selon Meinong, une telle affirmation doit être comprise en un sens métaphorique, car, pour lui, au sens strict, la seule réalité est celle du monde empirique $^{6}$. Cette réalité mondaine est avant tout temporelle, c'est-à-dire qu'est réel ce qui a existé, existe maintenant, ou existera dans le futur. De la sorte, la dernière neige qui vient de fondre est tout aussi réelle que la neige qui tombe actuellement ${ }^{7}$. Les objets fictifs 
ne sauraient être réels, parce tous les objets réels sont « complets » et que les objets fictifs sont, eux, fondamentalement « incomplets », comme nous le montrerons plus loin.

Si les objets fictifs ne sont pas réels, sont-ils pour autant doués d'un autre mode d'être? Refusant ce qu'il appelle "le préjugé en faveur de la réalité », Meinong fait une place à côté des objets réels aux « objets idéaux », c'est-à-dire aux objets qui, bien qu'ils puissent d'une certaine manière être affirmés, ne peuvent jamais être dits, sans commettre par là une erreur, "exister» (existieren), selon leur nature ${ }^{8}$. Si ces objets n'existent pas, ils peuvent toutefois «consister» (bestehen); la consistance étant, par opposition à l'existence, un mode d'être intemporel ${ }^{9}$. Parmi les objets idéaux, nous trouvons de manière exemplaire des objets mathématiques comme le triangle ou le nombre 5 . Meinong ne semble pas véritablement fournir d'argument en faveur de l'exclusion des objets fictifs du domaine de l'idéalité. Nous pourrions éventuellement dire que ces objets ne peuvent être idéaux, en raison du fait que certains d'entre eux sont contradictoires, que ce soit par négligence de leur créateur - parce celui-ci a par mégarde attribué à un même objet fictif des propriétés incompatibles - ou de manière délibérée, comme dans le cas du cercle-carré, et que les objets contradictoires sont dépourvus de tout mode d'être ${ }^{10}$

Meinong refuse donc que les objets fictifs puissent jouir d'un quelconque mode d'être. Pourtant, il reconnaît aussi qu'il est possible d'attribuer à ces objets des propriétés; ils possèdent ce qu'il appelle un "être-tel» (Sosein) ${ }^{11}$. Si nous pouvons attribuer des propriétés à ces objets, c'est parce qu'il y a un « principe d'indépendance de l'être-tel par rapport à l'être ${ }^{12}$. Autrement dit, un objet peut posséder des propriétés, même s'il est dépourvu de tout mode d'être. Par exemple, nous pouvons affirmer de manière vraie que le cercle-carré est rond, alors que le cercle-carré n'existe pas, et même n'est pas en aucun sens possible de ce terme. De manière générale, pour Meinong, les objets fictifs sont des objets qui possèdent des propriétés (c'est la condition minimale pour être un objet), tout en se voyant refuser un quelconque mode d'être. Ces objets sont alors dits « hors-être » ( außerseind), par-delà l'être et le non-être ${ }^{13}$.

Ingarden s'accorde avec Meinong pour dire que les objets fictifs ne sont ni réels ni idéaux ${ }^{14}$. Ils ne sauraient être réels, parce qu'ils sont incomplets, et ils ne sauraient être idéaux, parce qu'ils sont créés :

(...) Le Faust de Goethe a été créé à un moment déterminé. Nous pouvons même indiquer d'une manière relativement précise l'époque de sa création. Nous sommes également tous d'accord pour dire qu'il existe depuis sa création, même si nous comprenons mal ce que signifie ce discours sur son existence. ${ }^{15}$

Sur ce point, les opinions d'Ingarden et de Meinong diffèrent, puisque pour ce dernier les objets fictifs ne sont pas créés. Ils sont en quelque sorte déjà présents, disponibles parmi les objets hors-être ${ }^{16}$. Ce n'est là qu'une conséquence de l'adoption par Meinong de ce qu'il appelle « le principe de liberté illimitée d'assomption $»^{17}$ :

(...) Concernant chaque détermination propre ou, pour ainsi dire, ordinaire de

l'être-tel, il est en mon pouvoir, en vertu du principe de la liberté d'assomption, de

choisir, au moyen d'une intention appropriée, un objet auquel appartient en fait la détermination mentionnée. ${ }^{18}$

Soit les déterminations «rond» et "carré ». D’après le principe de liberté illimitée d'assomption, je peux par la pensée choisir un objet qui possède exactement ces déterminations, à savoir le cercle-carré, lequel objet, étant impossible, est situé en dehors de l'être. De même, je peux « choisir parmi l'infinie totalité des objets hors-être » un objet 
qui correspond exactement aux déterminations attribuées à Sherlock Holmes dans les œuvres de fiction écrites par Arthur Conan Doyle.

Pour Meinong, ce qui est créé, ce sont les mots et les phrases au moyen desquels un auteur vise les objets fictifs :

Ce que l'artiste "crée » [schafft] est une réalité plus ou moins composite, qui a la propriété, pour ceux qui l'appréhendent, de «signifier » quelque chose de plus ou moins composite, en particulier l'objet esthétique, qui de cette manière, pour ceux qui appréhendent cette réalité, est choisi parmi la totalité infinie des objets hors l'être. ${ }^{19}$

Lorsque Conan Doyle écrit un roman, il compose des expressions linguistiques qui expriment des représentations et des jugements ayant Sherlock Holmes pour objet. Mais, si Conan Doyle crée ces expressions, il ne crée pas l'objet qui est signifié par les phénomènes psychiques qu'elles expriment.

11 Ce point de divergence étant précisé, revenons à Ingarden. Pour lui, les objets fictifs ne peuvent être idéaux du fait qu'ils sont créés à un instant donné. Comment dès lors peuvent-ils être constitués par des phrases dont la signification est, pour sa part, idéale? Nous ne pouvons nier purement et simplement le caractère idéal de la signification des phrases, car ce serait retomber dans le psychologisme, si vigoureusement dénoncé par Husserl. La solution d'Ingarden passe ici par une reformulation complexe de la thèse de l'idéalité de la signification propositionnelle. En simplifiant quelque peu, nous pourrions dire qu'elle consiste à soutenir qu'une œuvre d'art littéraire est composée de significations, lexicales ou propositionnelles, qui ne sont pas idéales, mais qui « actualisent» (aktualisieren/aktualizują) des parties d'autres significations qui sont, quant à elles, idéales, et qu'Ingarden appelle des « concepts idéaux $»^{20}$. Cette actualisation est notamment réalisée lors de la formation d'une phrase ou d'un mot par un individu, par exemple l'auteur d'un texte littéraire.

12 L'objet fictif ne s'identifie ni au concept idéal, ni aux différentes significations lexicales qui actualisent ce dernier. Il est ce qui est visé par ces significations et qui exemplifie le concept idéal. De manière générale, l'effectuation de la visée de la signification " produit » (schafft/wytworzy), ou mieux «projette » (entwirft/sprojektuje) ou " figure » ( stellt dar/przedstawia) un objet, qu'Ingarden qualifie d'«intentionnel $»^{21}$. Cet objet intentionnel possède un mode d'être propre, ne se réduisant pas aux modes d'être réel et idéal : le mode d'être intentionnel.

13 Parmi les objets intentionnels, Ingarden distingue ceux qui sont « purement [rein/czysty] intentionnels» de ceux qui sont "aussi intentionnels» (auch intentional/także intencjonalny) $^{22}$. Par exemple, lorsque je lis dans un journal qu'une pipe a été retrouvée sur le lieu d'un crime, je vise intentionnellement cette pipe, qui est alors un objet aussi intentionnel, parce qu'elle n'est pas créée par ma visée, mais est ontologiquement « autonome », en un sens qu'il nous faudra préciser. Cette pipe existe indépendamment de tout acte intentionnel, mais peut également être visée par un acte intentionnel ${ }^{23}$. En revanche, lorsque je lis dans un roman de Conan Doyle que Sherlock Holmes fume la pipe, l'objet fictif correspondant au mot "pipe» est purement intentionnel, parce qu'il est simplement projeté ou figuré par la visée intentionnelle qui est activée en moi par la lecture.

14 Les objets fictifs issus de la littérature sont donc des objets projetés ou figurés par les significations qui composent le texte littéraire. Ces objets doivent être compris en un sens très large, puisqu'ils peuvent se rapporter à des personnes, des événements, des états de 
choses, etc. ${ }^{24}$ On parlera donc plus proprement «d'objectualités » (Gegenständlichkeiten) fictives ou de «corrélats objectuels» fictifs ${ }^{25}$. Ce sont des exemples typiques d'objectualités purement intentionnelles. Arrêtons-nous donc sur la définition que nous donne Ingarden de ces dernières :

Nous entendons par objectualité purement intentionnelle une objectualité qui est "créée ", en un sens dérivé, par un acte de conscience, respectivement par une multiplicité d'actes ou, enfin, par une formation (par exemple, une signification lexicale, une phrase), qui contient en soi l'intentionnalité empruntée, exclusivement sur la base d'une intentionnalité immanente originaire ou simplement empruntée, et qui a dans les objectualités mentionnées [à savoir l'acte de conscience, la multiplicité d'actes ou la formation] l'origine de son être [Seins/byt ] et de son être-tel [Sosein/uposażenie]. ${ }^{26}$

Les objectualités fictives étant des objectualités purement intentionnelles, l'éclaircissement du mode d'être de ces dernières nous renseignera directement sur le statut ontologique des Sherlock Holmes, Hamlet et autres Méphistophélès. Pour ne pas trop surcharger terminologiquement notre texte, nous parlerons dans ce qui suit simplement d'objets purement intentionnels, étant entendu que sous cette notion nous comprenons la notion d'objet au sens étendu d'objectualité.

\section{Le mode d'être des objets purement intentionnels selon Ingarden}

Dans sa troisième Logische Untersuchung, Husserl a développé une théorie pure des touts et des parties centrée sur la notion de "fondation $»^{27}$. Celle-ci est en fait une variété de dépendance existentielle au sens où un objet est fondé dans un autre lorsqu'il ne peut exister sans que cet autre objet existe également. Par exemple, une couleur ne peut exister sans une étendue recouverte par elle, et inversement. Ingarden, pour sa part, développe une analyse fouillée des différents types de dépendance existentielle, qu'il qualifie de «moments existentiels» (existentiale Momente/momenty bytowe). Il en identifie quatre paires opposées ${ }^{28}$ :

a) autonomie (Autonomie/autonomia) et hétéronomie (Heteronomie/heteronomia) ;

b) originarité (Ursprünglichkeit/pierwotność) et dérivabilité (Abgeleitetkeit/ pochodność) ;

c) séparabilité (Selbständigkeit/samodzielność) et inséparabilité (Unselbständigkeit/ niesamodzielność) ;

d) indépendance (Unabhängigkeit/niezależność) et dépendance (Abhängigkeit/zależność) 29 .

Ces moments existentiels se combinent alors pour former des "modes d'être" ( Seinsweisen/sposoby istnienia), comme ceux qui caractérisent les objets réels, les objets idéaux, ou encore les objets purement intentionnels. Examinons les différents moments existentiels qui caractérisent ce dernier mode d'être, puisqu'il s'agit de celui des objets fictifs.

16 Tout d'abord, les objets purement intentionnels sont hétéronomes ${ }^{30}$. Un objet est existentiellement hétéronome s'il possède un «fondement d'être " (Seinsfundament/ fundament bytowy) en dehors de lui-même ${ }^{31}$, qu'il n'est rien de spécifique en lui-même ${ }^{32}$. Dès lors, il n'a pas d'« essence propre » (Eigenwesen/własna istota), de nature qui lui soit totalement immanente. Par exemple, les caractéristiques de Sherlock Holmes - le fait qu'il s'agisse d'un homme, qu'il existe sur le mode de la réalité, etc. - ne lui sont qu'attribuées 
par Conan Doyle, il ne les possède pas en propre. Sherlock Holmes, s'il est projeté par une visée intentionnelle, n'est pas créé au sens strict, c'est-à-dire au sens où une fois créées par son auteur, les déterminations qui lui sont attribuées lui seraient « incorporées ». Le célèbre détective n'est qu'une " "apparence" qui puise dans l'intention projetante (...) de l'acte de visée le caractère illusoire de son être et de son être-tel $»^{33}$. Mais l'objet purement intentionnel qu'est Sherlock Holmes, s'il est un rien au sens de l'autonomie, puisqu'il ne peut ni être ni changer par lui-même, n'est pas pour autant un "néant total » (völliges Nichts/zupetne nic) ${ }^{34}$. Il s'agit bien d'un objet, ou mieux d'un "quasi-objet", quelque chose qui se donne " "comme un objet", "à la manière d'un objet", mais sans pouvoir prétendre rigoureusement à ce statut $»^{35}$.

L'opposition entre hétéronomie et autonomie, d'une part, et l'opposition entre inséparabilité et séparabilité, d'autre part, ne doivent pas être confondues ${ }^{36}$. Un objet $a$ est inséparable d'un objet $b \mathrm{si}$, d'après son essence, $a$ a nécessairement besoin, pour pouvoir être, de coexister avec $b$ au sein d'un tout ${ }^{37}$. Par exemple, une nuance particulière de rouge ne peut exister qu'au sein d'un objet dont elle est la couleur. Si elle est donc inséparable de l'objet dont elle est la couleur, elle n'est pas pour autant existentiellement hétéronome, puisqu'elle possède son fondement d'être en elle-même, qu'elle est en ellemême ce qu'elle est. Un objet fictif, en revanche, est hétéronome et séparable des actes psychiques qui le constituent au sens où, comme le dit Ingarden, il les «transcende ", c'est-à-dire qu'il n'en est pas une partie réelle ${ }^{38}$.

Les moments existentiels de dépendance et d'indépendance ne reviennent qu'aux objets séparables. Il est possible qu'un objet soit séparable et qu'il nécessite toutefois, en vertu de son essence, l'existence d'un autre objet séparable pour pouvoir lui-même continuer à être. Le premier objet est alors dit existentiellement dépendant du second ${ }^{39}$. Les objets fictifs littéraires sont des entités dépendantes en ce sens, puisqu'ils ont besoin pour pouvoir se maintenir dans l'être de l'existence d'un autre objet: leur créateur ou un lecteur.

Dans L'CEuvre d'art littéraire, Ingarden distingue, parmi les objets qui sont purement intentionnels, ceux qui le sont « de manière originelle » (ursprünglich/pierwotnie) et ceux qui le sont " de manière dérivée » (abgeleitet/wtórnie) ${ }^{40}$. Les premiers " puisent l'origine de leur être et de leur être-tel directement dans les actes concrets de conscience posés par un moi ». Les objets imaginés constituent des objets purement intentionnels de manière originelle en ce sens, puisqu'ils tirent leur nature et leur être entièrement des actes d'imagination qui les ont produits. Ce n'est pas pour autant que ces objets sont immanents à la conscience, que ce sont des parties réelles des actes qui les produisent. Ils sont bien distincts de ces actes, tout en en étant totalement dépendants. Les objets purement intentionnels de manière dérivée, quant à eux, doivent leur être et leur être-tel à des formations " qui contiennent en elles une intentionnalité "empruntée" " ${ }^{41}$. C'est par exemple le cas des objets fictifs littéraires ${ }^{42}$. Ceux-ci ne tirent pas leur être et leur être-tel directement d'actes de conscience, mais des significations qui sont exprimées par les phrases qui composent le texte littéraire, lesquelles significations renvoient à l'intentionnalité originelle des actes de conscience de l'auteur.

L'œuvre d'art littéraire ne se réduit pas à son support matériel, par exemple un texte écrit; elle en est indépendante. Ce support est plutôt ce qui « lui assure une certaine permanence et surtout l'ouvre à la possibilité d'une réception et d'une appropriation par d'autres sujets que le sujet créateur $\aleph^{43}$. Ainsi, les objets purement intentionnels de manière dérivée ont un avantage insigne sur ceux qui le sont de manière originelle : 


\begin{abstract}
Alors que ces derniers sont des formations "subjectives ", en ce sens que leur caractère originel leur vaut de n'être immédiatement accessibles que par un sujet de conscience, [à savoir par] celui qui a opéré leur "création ", et d'avoir aux actes concrets une relation d'appartenance irrévocablement nécessaire, les premiers, en tant que corrélats d'unités de signification, sont intersubjectifs : ils peuvent être visés, ou saisis comme identiquement les mêmes par différents sujets de conscience.

44
\end{abstract}

21 C'est « l'intentionnalité d'emprunt » conférée par l'auteur aux mots qui composent un texte littéraire qui permet un accès intersubjectif aux objets purement intentionnels de manière dérivée que sont les objets fictifs, là où les objets qui sont purement intentionnels de manière originelle ne sont accessibles qu'à celui qui les a créés.

La distinction, que l'on trouve dans L'Cuvre d'art littéraire, entre les objets qui sont purement intentionnels de manière dérivée et ceux qui le sont de manière originelle n'est pas identique à la distinction entre les moments existentiels d'originarité et de dérivabilité, du moins telle qu'elle est formulée dans Spór o istnienie świata (La Controverse sur l'existence du monde, 1947-1948) ${ }^{45}$. Dans ce deuxième texte, Ingarden définit un objet comme étant existentiellement dérivé si, d'après son essence, il peut être produit par un autre objet ${ }^{46}$. Les objets purement intentionnels, et en particulier les objets fictifs, sont des objets dérivés en ce sens, puisqu'il est dans leur essence de devoir être produits par d'autres objets pour pouvoir exister. Selon Ingarden, les objets existentiellement originels "contiennent la source de leur être en eux-mêmes", de sorte qu'ils sont existentiellement durables ; ils ne peuvent ni être créés ni être détruits par un autre objet 47 .

Il y a donc des objets qui possèdent une " propension à continuer à subsister ${ }^{48}$. Mais il y en a aussi d'autres qui cessent d'exister s'ils ne sont pas «maintenus » dans leur être par celui qui les a créés ou par un autre objet. Tandis que les premiers continuent à exister grâce à leur « inertie existentielle » (Tragheit des Seins/bezwładność istniena) ${ }^{49}$, les seconds en sont dépourvus. L'analogie trouve ici clairement son origine dans le domaine physique: de même que, selon le principe d'inertie de la mécanique newtonienne, un corps persévère dans son mouvement rectiligne uniforme ou dans son état de repos, à moins qu'il ne se trouve soumis à une force qui le contraigne à changer d'état, selon le principe ontologique d'inertie, certains objets persévèrent dans leur mode d'être. Ainsi, pour reprendre les exemples d'Ingvar Johansson ${ }^{50}$, les objets idéaux ont une inertie existentielle au sens le plus fort possible, puisqu'ils ne peuvent cesser d'être. Les objets réels, quant à eux, persistent dans leur mode d'être à moins que quelque chose d'autre ne les en fasse sortir. Finalement, les objets fictifs, étant hétéronomes et dérivés, sont dépourvus de toute inertie existentielle au sens où ils ne persévèrent pas d'eux-mêmes dans leur mode d'être, celui-ci doit être soutenu par une autre entité. Autrement dit, si personne ne pense à Hamlet, celui-ci cesse d'être. On pourrait ici évoquer le roman de Jean Ray, Malpertuis, dans lequel les dieux grecs meurent lorsque les hommes cessent de croire en eux.

\title{
L'incomplétude des objets fictifs
}

Barack Obama est un objet réel. De ce point de vue, il est a priori possible de dire de toute propriété s'il la possède ou s'il possède la propriété complémentaire. Je ne sais pas combien de cheveux il possède, mais même si je ne peux le déterminer en pratique, cela n'est pas impossible en droit. Indépendamment de mon ignorance concernant le nombre 
de cheveux de Barack Obama, il est certain que cet individu possède un nombre déterminé de cheveux et que, ceci étant admis, soit il possède un nombre pair de cheveux, soit il en possède un nombre impair. Considérons à présent l'inspecteur Lestrade. Il n'y a pas de fait dans les romans de Conan Doyle nous indiquant le nombre de cheveux qui peuplent son crâne, de sorte que, étant donné qu'il a des cheveux, il n'est ni le cas qu'il en possède un nombre pair, ni qu'il en possède un nombre impair. À la différence de l'indétermination qui concernait Barack Obama, celle qui concerne l'inspecteur Lestrade n'est pas de nature épistémologique, mais bien ontologique ${ }^{51}$. Le premier type d'indétermination est dû à un manque d'information concernant un individu réel, alors que celle du deuxième type est relative à un objet fictif et ne pourra jamais être comblée par une quelconque information supplémentaire, elle lui est intrinsèque: nous ne pouvons savoir si le nombre de cheveux de l'inspecteur Lestrade est pair ou impair, parce que Conan Doyle ne l'a pas précisé, et non parce que nous avons oublié ou manqué cette information dans le texte.

D’après Meinong, Barack Obama est un objet "complet» (vollständig), tandis que l'inspecteur Lestrade est un objet « incomplet». Un objet est complet, lorsque, de toute détermination (positive ou négative), nous pouvons dire qu'elle lui convient ou qu'elle ne lui convient $\mathrm{pas}^{52}$. Selon Meinong, tout objet réel est complet en ce sens. Les objets fictifs en revanche sont toujours incomplets. Mais ce n'est pas le seul exemple d'objets de ce type. Par exemple, les « objets conceptuels » (Begriffsgegenstände), comme le triangle, sont également incomplets. Cet objet ne possède ni ne possède pas certaines propriétés; entre autres, l'équilatéralité. Si nous pouvons dire de tout triangle particulier s'il est équilatéral ou non, ce n'est pas le cas du triangle pris en général.

Les objets incomplets ne possédant ni ne possédant pas certaines propriétés, il semble qu'ils ne satisfont pas le principe du tiers exclu. En fait, tout dépend de la manière dont nous énonçons ce principe. Dans la formulation suivante, que nous pouvons qualifier d' ontologique :

(1) $a$ possède $F$ ou $a$ ne possède pas $F$, la négation est ambiguë. Porte-t-elle sur le prédicat $F$ ou sur la proposition a possède $F$ dans sa totalité ? Est-elle nominale ou propositionnelle? Meinong distingue clairement ces deux sens de la négation: il appelle "étendue " la négation qui porte sur toute la proposition et « restreinte » celle qui porte uniquement sur le prédicat ${ }^{53}$.

En prenant la négation figurant dans (1) au sens restreint, nous obtenons alors la formulation suivante du principe ontologique du tiers exclu :

(2) $a$ possède $F$ ou $a$ possède non- $F$, que nous qualifierons de restreinte. Les objets incomplets ne satisfont pas ce principe ${ }^{54}$. En revanche, ils satisfont la formulation étendue du principe ontologique du tiers exclu :

(3) $a$ possède $F$ ou il n'est pas le cas que $a$ possède $F$. Qu'en est-il à présent de ce que Jan Łukasiewicz appelle le « principe ontologique de [non]contradiction $»^{55}:$ « aucun objet ne peut à la fois posséder et ne pas posséder une même propriété »? Tous les objets fictifs satisfont la version de ce principe dans laquelle la négation est comprise en un sens étendu. En ce qui concerne la version restreinte, tout dépend du type d'objet fictif auquel nous avons affaire. L'inspecteur Lestrade, par exemple, est un objet fictif possible qui satisfait la version restreinte de ce principe. Mais nous pouvons tout à fait concevoir des objets fictifs qui, comme le cercle-carré, sont impossibles, du fait qu'ils possèdent des propriétés contradictoires, et qui pour cette raison ne satisfont pas la version restreinte du principe ontologique de non-contradiction. 
Ingarden oppose lui aussi les objets réels et les objets fictifs en termes d'incomplétude : tandis que les premiers satisfont le principe ontologique du tiers exclu ${ }^{56}$, les seconds sont des «formations schématiques" qui présentent ce qu'il appelle des «lieux d'indétermination" (Unbestimmtheilsstellen/miejsca niedookreślenia) ${ }^{57}$. Par exemple, la couleur des yeux de Sherlock Holmes est indéterminée, tout simplement parce que Conan Doyle n'a pas créé son personnage avec cette propriété. Cela ne veut pas dire que ces lieux d'indétermination soient accidentels, au sens où ils résulteraient d'une erreur de composition de la part de l'auteur. Au contraire, leur présence est nécessaire dans toute œuvre d'art littéraire, puisqu'avec un nombre fini de mots et de phrases, et donc de significations, on ne peut projeter de manière exhaustive le nombre infini de déterminations requises pour compléter intégralement un objet fictif ${ }^{58}$. L'auteur peut toujours préciser tel ou tel aspect indéterminé, mais il ne peut, par essence, les compléter tous.

Ingarden va plus loin que Meinong dans sa reconnaissance d'une possibilité de remplissage intentionnel par le lecteur des lieux d'indétermination inhérents aux œuvres d'art littéraires :

(...) Le lecteur, lors de la lecture et de la saisie esthétique de l'œuvre, dépasse le donné textuel (respectivement ce que projette le texte) et complète à de nombreux égards les «objectualités » figurées (...); de sorte qu'au moins certains lieux d'indétermination sont éliminés, souvent comblés par des déterminations que non seulement le texte ne détermine pas, mais qui ne s'accordent pas non plus avec les moments objectuels positivement déterminés par celui-ci. ${ }^{59}$

Ce remplissage des lieux d'indétermination lors de la lecture, Ingarden l'appelle " concrétisation » (Konkretisation/konkretyzacja). C'est grâce à elle que je visualise Sherlock Holmes avec des yeux bleus, même si la couleur de ses yeux n'est pas précisée par le texte écrit par Conan Doyle.

Une même œuvre littéraire peut à l'évidence être concrétisée de différentes manières. Par exemple, la couleur que j'attribue aux yeux de Sherlock Holmes lors de ma lecture n'est pas forcément la même que celle que lui attribuera un autre lecteur, du moins s'il lui en attribue une. Ces multiples concrétisations donnent en quelque sorte "vie » à l'œuvre d'art littéraire, " une "vie historique" de l'œuvre qui se poursuit par-delà l'intention de son créateur au moyen des réactivations intentionnelles des sujets récepteurs ${ }^{60}$. L'œuvre projetée par le texte littéraire, dit Ingarden, est comme un «squelette », qui revêt différents traits et détails dans la concrétisation, comme le ferait un «corps vivant $»^{61}$. Le squelette est alors ce qui, visible sous le corps de l'œuvre concrétisée, lui assure son identité (intersubjective).

\section{Deux types de propriétés ou de prédications?}

Les principes de liberté illimitée d'assomption et d'indépendance de l'être par rapport à l'être-tel permettent à Meinong d'inclure dans sa théorie des objets non existants comme la montagne d'or ou le cercle-carré. Ils lui permettent en fait d'y inclure tout objet correspondant à un ensemble arbitraire de propriétés. Qui plus est, tout objet généré de la sorte possède les propriétés qui le caractérisent. Nous pouvons ainsi affirmer que la montagne d'or est dorée ou que le cercle-carré est rond. Russell a vu dans cette caractéristique de la théorie meinongienne de l'objet la source d'une contradiction susceptible de l'ébranler ${ }^{62}$. Qu'en est-il en effet de l'objet correspondant à l'ensemble \{être 
rond, être carré, exister\}, c'est-à-dire du cercle-carré existant ? Tout objet qui possède à la fois la propriété d'être rond et d'être carré est impossible, et donc non existant. En même temps, il semble analytiquement vrai que le cercle-carré existant existe. Il y a donc une contradiction : le cercle-carré existant à la fois existe et n'existe pas.

C'est pour échapper à ce type de paradoxe que certains néomeinongiens, comme Parsons ${ }^{63}$, ont proposé de s'appuyer sur une distinction, due originellement à Ernst Mally, entre propriétés "nucléaires » et " extranucléaires " ${ }^{64}$. La solution consiste alors à dire que les seules propriétés avec lesquelles nous pouvons générer un objet au moyen du principe de liberté illimitée d'assomption sont les propriétés nucléaires et que l'existence n'est précisément pas une propriété de ce type, mais une propriété extranucléaire.

Les propriétés nucléaires sont celles auxquelles font référence les prédicats dans les phrases :

(4) Sherlock Holmes habite au 221B Baker Street;

(5) Le docteur Watson a été médecin en Afghanistan, etc.,

tandis que les propriétés extranucléaires sont celles auxquelles font référence les prédicats dans les phrases :

(6) Sherlock Holmes est un personnage fictif ;

(7) Sherlock Holmes est le détective privé préféré d'Agatha Christie, etc.

Une manière de caractériser cette différence, dans le cas des objets fictifs, serait de dire que la vérité des phrases du premier type est conditionnelle, au sens où ce qu'elles affirment n'est pas vrai de manière absolue, mais d'après ce qui est dit dans les romans de Conan Doyle. Dès lors, il semblerait que lorsque nous affirmons que Sherlock Holmes habite au 221B Baker Street, ce que nous voulons en fait dire, c'est que : d'après ce qui est dit dans les romans de Conan Doyle, Sherlock Holmes habite au 221B Baker Street. En revanche, une analyse de ce type ne fonctionne pas pour les phrases du second type (celles qui contiennent une référence à une propriété extranucléaire). Par exemple, d'après de ce qui est dit dans les romans de Conan Doyle, Sherlock Holmes n'est pas un personnage fictif, c'est un individu tout aussi réel que le docteur Watson.

Nous retrouvons également chez Ingarden cette idée selon laquelle deux types de propriétés différentes peuvent échoir aux objets purement intentionnels, et donc aux objets fictifs. Selon lui, les objets ont un double aspect, ils sont bifaces (doppelseitig/ dwustronny). Plus précisément, ils possèdent à la fois une "teneur " (Gehalt/zawartość) et une "structure " $(\text { Struktur/struktura })^{65}$. Les propriétés qui reviennent à la teneur sont analogues aux propriétés nucléaires de Parsons, ce sont les déterminations projetées par le texte littéraire, tandis que les propriétés qui reviennent à la structure sont analogues aux propriétés extranucléaires, ce sont les déterminations qui reviennent à l'objet en tant qu'objet intentionnel. L'objet fictif semble ainsi se dédoubler en deux "porteurs" de propriétés distincts - il y a, dit Ingarden, « une étrange dualité, un curieux "double supportement" [Doppelträgerschaft/dwupodmiotowość]» de l'objet fictif. Par exemple, Sherlock Holmes se donne, d'une part, comme un détective réel, ami du docteur Watson et, d'autre part, comme un objet fictif inventé par Conan Doyle et apprécié par Agatha Christie.

\section{Conclusion}

Cette comparaison des approches ingardénienne et meinongienne des objets fictifs montre bien leur étroite proximité de départ, en particulier en ce qui concerne la 
dimension ontologico-formelle de ces objets. En effet, Meinong et Ingarden conçoivent tous deux les objets fictifs comme des objets incomplets auxquelles échoient deux types de propriétés différentes. Ingarden va néanmoins plus loin que Meinong sur ce point, envisageant la possibilité d'une concrétisation des objets fictifs lors de la lecture.

C'est du point de vue ontologico-existentiel, que les positions de nos deux auteurs divergent le plus profondément. Car s'ils s'accordent pour dire que les objets fictifs ne sont ni réels ni idéaux, ce n'est pas le cas en ce qui concerne le mode d'être qui devrait alors leur revenir. Cette divergence a une conséquence fondamentale sur leur approche générale de la fiction: tandis qu'Ingarden met l'accent sur les différents types de dépendance qui échoient aux objets fictifs en vertu de leur création par un sujet conscient, Meinong souligne leur indépendance. En effet, pour ce dernier, l'objet ne saurait en général être créé par le sujet. Si le sujet conscient «saisit» ou «appréhende » (erfasst) l'objet, en aucun cas, il ne le constitue :

Face à l'appréhension, son objet est chaque fois ce qui logiquement est antérieur, même lorsque cet objet suit chronologiquement l'appréhension. C'est pourquoi l'appréhension ne peut jamais créer son objet ou ne serait-ce que le modifier mais simplement le sélectionner en quelque sorte, en l'extrayant de la diversité de ce qui est préalablement donné (du moins comme étant hors l'être). ${ }^{66}$

L'objet a une consistance propre, une essence, qui peut être appréhendée par le sujet, mais qui est indépendante de lui. L'auteur qui crée une œuvre de fiction ne l'extrait en fait que du domaine de l'hors-être, domaine qui, comme son nom l'indique, ne relève pas de l'être, mais du simple il y a (es gibt) qui caractérise tout objet. Ainsi, là où Ingarden attribuait aux objets fictifs un mode d'être propre - celui des objets purement intentionnels, hétéronomes, dérivés, séparables et dépendants - Meinong leur refuse tout mode d'être, les situant au-delà de l'être et du non-être. La description ontologique d'Ingarden paraît à cet égard bien supérieure à celle de Meinong : les objets fictifs ne sont pas simplement déjà là, prêts à être ramassés par le premier ontologue venu qui parcourrait le domaine existentiellement neutre de l'hors-être ; ils sont doués d'un mode d'être qui leur est propre et qui les distingue de tous les autres types d'objets, qu'ils soient réels, idéaux ou même divins.

\section{NOTES}

1. Elle a notamment été abordée par Barry Smith: "Ingarden vs. Meinong on the Logic of Fiction» (Ingarden contre Meinong sur la logique de la fiction), in: Philosophy and Phenomenological Research, t. XLI, cahiers 1-2, 1980, pp. 93-105.

2. Raspa V., "Fictional and Aesthetic Objects: Meinong's Point of View " (Objets fictifs et esthétiques: le point de vue de Meinong), in: Modes of Existence (Modes d'existence), sous la direction de Bottani A. \& Davies R., Ontos, Francfort, 2006, p. 47.

3. On trouve également une approche de type néo-ingardénienne dans la philosophie contemporaine de la fiction chez Amie L. Thomasson: Fiction and Metaphysics (Fiction et Métaphysique), Cambridge University Press, Cambridge, 1999. 
4. Pour une biographie des travaux sur les logiques néomeinongiennes, cf. Leclercq Br., «Faire cohabiter les objets sans domicile fixe (homeless objects): Chisholm et les logiques meinongiennes ", in : Bulletin d'analyse phénoménologique, t. X, cahier 6, 2014, n. 2, p. 89.

5. Lipps Th., « Weiteres zur "Einfühlung" » (Plus d'information sur «l'empathie»), in : Archiv für die gesamte Psychologie, t. IV, 1905, pp. 487-488.

6. Meinong A., «Über Urteilsgefühle: was sie sind und was sie nicht sind » (Sur les jugements de sensation: ce qu'ils sont et ce qu'ils ne sont pas), in: Meinong A., Gesamtausgabe (Æuvres complètes), vol. I, Akademische Druck u. Verlagsanstalt, Graz, 1969, p. 599.

7. Meinong A., « Sur les objets d'ordre supérieur et leur rapport à la perception interne », traduit de l'allemand par Fréchette G., in: À l'école de Brentano, sous la direction de Fisette D. \& Fréchette G., Vrin, Paris, 2007, § 21, pp. 330-331.

8. Ibid., § 6, p. 275.

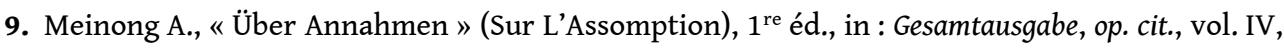
1977, § 41, p. 467.

10. Il leur échoit au mieux un mode de non-être.

11. Meinong A., "La Théorie de l'objet », traduit de l'allemand par J-Fr. Courtine et M. de Launay, in : Meinong A., Théorie de l'objet et Présentation personnelle, Vrin, Paris, 1999, § 3, p. 71.

12. Ce principe fut formulé pour la première fois en 1903 par Ernst Mally, un élève de Meinong.

13. Meinong A., « La Théorie de l'objet », art. cit., § 4, p. 76.

14. Pour Roman Ingarden également, les objets réels et idéaux se distinguent d'un point de vue temporel. Tandis que les premiers sont "produits à un moment [donné] », ont " une certaine durée », se modifient « éventuellement pendant ce temps » et cessent « finalement d'exister », les seconds sont « atemporels» (zeitlos/bezczasowy). Cf. Ingarden R., L'Euvre d'art littéraire, traduit de l'allemand par Ph. Secretan, L'Âge d'homme, Lausanne, 1983, § 3, p. 27. La question de la langue originale dans laquelle Ingarden a écrit ses textes étant passablement complexe, nous indiquons systématiquement entre parenthèses les termes allemands et polonais.

15. Ibid., § 3, pp. 27-28.

16. Meinong A., «Über Annahmen », $2^{e}$ éd, in : Gesamtausgabe, op. cit., vol. IV, § 45, p. 274.

17. Ibid., § 60, p. 346 .

18. Meinong A., «Möglichkeit und Wahrscheinlichkeit» (Possibilité et Probabilité), in: Gesamtausgabe, op. cit., vol. VI, 1972, § 37, p. 282.

19. Meinong A., "Über Urteilsgefühle: was sie sind und was sie nicht sind », in : Gesamtausgabe, op. cit., vol. I, p. 603.

20. Ingarden R., L'Eeuvre d'art littéraire, op. cit., § 16, p. 88.

21. Ibid., § 15, p. 72.

22. Ibid., § 20, p. 111 (traduction modifiée).

23. Johansson I., "Fictions and the Spatiotemporal World - in the Light of Ingarden" (Les Fictions et le monde spatiotemporel - à la lumière d'Ingarden), in : Polish Journal of Philosophy, t. IV, cahier 2, 2010, p. 92.

24. Ingarden R., L'CEuvre d'art littéraire, op. cit., § 32, p. 189.

25. Sur la notion d'objectualité, nous nous permettons de renvoyer à : Richard S., De la forme à l'être, Ithaque, Montreuil, 2014, pp. 209 et suiv.

26. Ingarden R., L'Euvre d'art littéraire, op. cit., § 20, p. 111 (traduction modifiée).

27. Cf. la troisième Recherche logique, in : Husserl E., Recherches logiques. Tome 2, vol. II., traduit de l'allemand par H. Elie et al., 4 éd., PUF, Paris, 2002, pp. 5-81; et pour une analyse : Richard S., op. cit., chap. 6.

28. Ingarden R., Spór o istnienie świata (La Controverse sur l'existence du monde), vol. I, $2^{\mathrm{e}}$ éd., PWN, Varsovie, 1960, § 10, p. 93 [§ 11, p. 78]. Pour cet ouvrage, nous indiquons les références à la version allemande (rédigée par Ingarden lui-même) entre crochets : Ingarden R., Der Streit um die Existenz der Welt, 3 vol., Niemeyer, Tübingen, 1964-1974. Ingarden analyse les différents moments 
existentiels dans les §§ 11-14 du Spór (§§ 10-13 de la version allemande), mais aussi dans: Ingarden R., "Remarques sur le problème "idéalisme-réalisme" ", traduit de l'allemand par P. Limido-Heulot, in : Ingarden R., La Controverse idéalisme-réalisme, Vrin, Paris, 2001, pp. 174 et suiv.

29. Afin d'éviter une répétition un peu lourde, nous avons omis l'expression « de l'être » (seins-/ bytowa), que nous aurions aussi pu traduire de manière moins exacte mais plus élégante par «existentiel » ou par «ontologique», et qui devrait suivre chaque expression des moments existentiels. Ainsi, la première paire n'oppose pas l'autonomie à l'hétéronomie, mais bien l'autonomie de l'être (Seinsautonomie/bytowa autonomia) à l'hétéronomie de l'être ( Seinsheteronomie/bytowa heteronomia).

30. Ingarden R., L'Euvre d'art littéraire, op. cit., § 21, p. 117.

31. Ingarden R., Spór o istnienie świata, op. cit., I, § 11, p. 94 [§ 12, p. 79].

32. Ingarden R., « Remarques sur le problème "idéalisme-réalisme” ", art. cit., p. 174.

33. Ingarden R., L'Eeuvre d'art littéraire, op. cit., § 20, p. 115 (traduction modifiée).

34. Ingarden R., Spór o istnienie świata, op. cit., I, § 11, p. 99 [§ 12, p. 84].

35. Limido-Heulot P., "Phénoménologie et ontologie chez Roman Ingarden », in : Ingarden R., La Controverse idéalisme-réalisme, op cit., p. 61.

36. Ingarden R., Spór o istnienie świata, op. cit., I, § 13, p. 138 [§ 14, p. 120].

37. Avec cette notion d'inséparabilité, Ingarden ne fait que reformuler celle, husserlienne, de fondation, que nous mentionnions plus haut.

38. Ingarden R., L'Eeuvre d'art littéraire, op. cit., § 20, p. 112 (traduction modifiée).

39. Ingarden R., Spór o istnienie świata, op. cit., I, § 14, pp. 138-139 [§ 15, p. 121].

40. Ingarden R., L'CEuvre d'art littéraire, op. cit., § 20, p. 111.

41. Idem.

42. Cela s'applique en fait aux œuvres d'art en général, mais aussi aux objets culturels, comme l'argent, les églises et les drapeaux.

43. Limido-Heulot $P$., «L'œuvre architecturale est-elle un objet intentionnel ? ", in : Roman Ingarden. La Phénoménologie à la croisée des arts, sous la direction de Limido-Heulot P., Presses Universitaires de Rennes, Rennes, 2013, p. 51.

44. Ingarden R., L'Euvre d'art littéraire, op. cit., § 21, p. 118 (traduction modifiée).

45. Ils diffèrent ne serait-ce que parce que tout autant les objets qui sont purement intentionnels de manière dérivée que ceux qui le sont de manière originelle peuvent posséder les deux moments existentiels en question.

46. Ingarden R., Spór o istnienie świata, op. cit., I, § 12, p. 112 [I, § 13, p. 87].

47. Ingarden R., « Remarques sur le problème "idéalisme-réalisme” », art. cit., p. 175.

48. Ingarden R., Spór o istnienie świata, vol. III, traduit de l'allemand par D. Gierulanka, PWN, Varsovie, 1981, p. 85.

49. Ingarden R., Spór o istnienie świata, op. cit., I, § 12, p. 130 [§ 13, p. 113].

50. Johansson I., "The Basic Distinctions in Der Streit» (Les Distinctions de base dans La Controverse), in : Semiotica, t. CXCIII, 2013, pp. 142-143.

51. Smith B., art. cit., p. 97.

52. Meinong A., "Présentation personnelle», traduit de l'allemand par J.-Fr. Courtine et M. de Launay, in : Meinong A., Théorie de l'objet..., op. cit., B. II, p. 136.

53. Meinong A., Möglichkeit und Wahrscheinlichkeit, op. cit., § 25, p. 171.

54. Pour une présentation plus détaillée, nous renvoyons à : Richard S., op. cit., pp. 166-168.

55. Łukasiewicz J., Du Principe de contradiction chez Aristote, traduit du polonais par D. Sikora, L'éclat, Paris, 2000, p. 48.

56. Ingarden R., « Remarques sur le problème "idéalisme-réalisme” », art. cit., p. 182.

57. Ingarden R., L'CEuvre d'art littéraire, op. cit., § 38, p. 211. 
58. Ingarden R., O poznawaniu dzieła literackiego (Sur La Connaissance de l'œuvre d'art littéraire), in : Ingarden R., Studia z estetyki, vol. I, $2^{\mathrm{e}}$ éd., PWN, Varsovie, 1966, § 11, p. 40.

59. Ingarden R., L'CEuvre d'art littéraire, op. cit., § 38, p. 214 (traduction modifiée).

60. Limido-Heulot P., « L'œuvre architecturale est-elle un objet intentionnel ?», art. cit., p. 53.

61. Ingarden R., L'Euvre d'art littéraire, op. cit., § 2, n. 1, p. 26.

62. Russell B., " Review of Untersuchungen zur Gegenstandstheorie und Psychologie » (Compte-rendu des Recherches sur la théorie de l'objet et la psychologie), in : Mind, t. XIV, cahier 56, 1905, p. 533.

63. Parsons T., «A Meinongian Analysis of Fictionnal Objects» (Une analyse meinongienne des objets fictifs), in : Grazer philosophische Studien, t. I, 1975, p. 76.

64. Cf. Jacquette D., «Nuclear and Extranuclear Properties» (Propriétés nucléaires et extranucléaires), in : The School of Alexius Meinong, sous la direction de Albertazzi L. et al., Ashgate, Aldershot, pp. 397-426.

65. Ingarden R., L'Euvre d'art littéraire, op. cit., § 20, p. 112 (traduction modifiée).

66. Meinong A., « Présentation personnelle », art. cit., F. II, p. 167.

\section{INDEX}

Mots-clés : Ecole de Brentano, Ingarden Roman, Meinong Alexius, phénoménologie, philosophie

\section{AUTEUR}

\section{SÉBASTIEN RICHARD}

Chargé de recherches du FNRS - Université Libre de Bruxelles 